\title{
Deployment of an Energy Efficient Routing Protocol for Wireless Sensor Networks Operating in a Resource Constrained Environment
}

\author{
Agbotiname Lucky Imoize ${ }^{1,2}$, Taiwo Oyedare ${ }^{1}$, Chibuike Gerald Ezekafor ${ }^{2}$, and Sachin Shetty ${ }^{3}$ \\ ${ }^{1}$ Bradley Department of Electrical and Computer Engineering, Virginia Tech, Blacksburg, USA \\ ${ }^{2}$ Department of Electrical and Electronics Engineering, University of Lagos, Akoka Lagos, Nigeria \\ ${ }^{3}$ Virginia, Modeling, Analysis and Simulation Center, Old Dominion University, Norfolk, VA, USA \\ aimoize@vt.edu, toyedare@vt.edu, jralde@yahoo.com, sshetty@odu.edu
}

\begin{abstract}
Wireless Sensor Networks often exist in a resource constrained environment and the most critical limitation is energy or battery life. In most cases, the batteries cannot be recharged during operation and cannot be replaced during transmission without interfering with the quality of service. An efficient routing protocol that can prolong the life span of the energy source for mobile wireless sensor network (WSN) is proposed in this paper. Hierarchical routing protocol improved the life span by more than six times compared to direct techniques. Sets of varied parameters were used to obtain simulation results. A sample typical of a hospital layout was used in this paper. Wards were assigned varied sensor range which depicts number of patients that fall into these banded regions of clusters. Service centers were created to ensure that random mobilization of sensors or patient per round is achieved for one of the simulation option. Simulation rounds were carried out for non-hierarchical (direct) and low energy adaptive clustering hierarchy (LEACH) based routing protocols. Model validation was done by setting up a real life test using Wi-Fi sensor node module ESP8266 properly distributed, and this was done without mobility. Effect of base station location was also investigated and results tabulated. With improved battery and network life span from both simulation and live validation for hierarchical routing, quality of service is greatly improved in health-care deliveries; cost of replacing energy sources is reduced. Results were also compared with other similar works to support our conclusion. Though results were not exact when compared, parameters used could cause tolerable difference. Furthermore, same result could not be achieved for different locations, varied number of wards, varied number of sensor nodes per wards as well as variations in other modeling parameters.
\end{abstract}

Keywords: Energy efficient routing protocol, Wireless sensor network, Adaptive clustering, Healthcare, Base station.

\section{Introduction}

The revolution caused by the emergence of wireless sensor technology (WST) cannot be over emphasized. The trend is quite significant and the application is almost limitless. Researchers have consistently explored this field of great interest and new grounds and methodologies have been identified [1]-[2]-[3][4] thereby making the challenges of today success stories of tomorrow. Wireless sensor network DOI: 10.14738/tnc.71.5975

Publication Date: 02 ${ }^{\text {nd }}$ February 2019

URL: http://dx.doi.org/10.14738/tnc. 71.5975 
application used to be a stationary based technology. With the demands for real time control and monitoring in various areas of interests, mobile wireless sensor network became a necessity. Our world today is enjoying the great dividends of wireless sensor networks, and more useful applications of WSNs are envisaged in the near future.

Telemedicine, military surveillance, industrial and environmental monitoring and other areas of application have become popular today. The value added to both life expectancy and other nature's course is quite notable. Wireless body sensor network mostly applied for medical information monitoring and control process is designed as a wearable system or implanted into the human body parts. The fact that sensor nodes need longer battery life to sustain the wireless sensor activities and the maintenance routine required when these sensors approach the end of their lifetime is of great concern. It is also more challenging to manage this in a mobile wireless sensor network [5]. Cost is always a key factor in approaches and models, hence, it is very imperative to explore this area and come up with a model that attempts to proffer an acceptable solution on how energy source can be optimized to extend wireless sensor network (WSN) lifetime. In this medical application, the role of sensors is to obtain vital signs and intelligently relay them to designated receiver or receiving node. In essence, a standard sensor node for this function consists of four major sections; the sensing unit, communication unit, processing section and the power unit. All these sections work seamlessly to achieve a quality throughput.

However, there are lots of limitations and performance/system integrity threat currently affecting the deployment and maximal usage of WSN. These WSNs have stern resource constraints and energy conservation cannot be overemphasized [6]. Since all these building blocks/units of the sensor node rely greatly on power to function, it implies that constant energy availability and sustainability is a major threat to wireless body sensor node network [7]. There has been various works on improving on the energy efficiency of each sensor node. All these are aimed at either reducing the power consumption of the network or increasing the battery life span. Local processing by nodes has also been considered though this implies complex circuitry and more power consumption. Adding mobility to the WSN system increases the complexity as it becomes more challenging to achieve maximum energy conservation or power efficiency. Amongst many other energy optimization methods, Hierarchical routing model has proven to present a better result. Hierarchical routing technique involves creating clusters for sensor nodes, assigning sensor nodes to cluster zone depending on the parameter used, and then selecting a cluster head which helps to obtain data from members of the same cluster for relay to other cluster heads or base station (BS). In this scenario, energy depletion on the network is reduced as well as service cost.

This study focuses on this technology and the methodology applied in this model. It also highlights few other challenges peculiar to mobile sensor nodes. Furthermore, this study is useful in deciding the best cluster formation for an health care environment before the deployment of mobile sensor nodes technology in such an environment. One should note however, that the best cluster formation for one environment may not necessarily be the best in another environment. This study therefore works with a typical predetermined environmental layout. In practice however, the layout parameters within this study will have to be re-defined for the environment under study.

The organization of this paper is as follows. Section II presents the literature survey of related works on the challenges with WSN and applications, routing protocols based on flat, location and hierarchy model and general challenges. Section III provides a theoretical background for routing protocol challenges as 
Agbotiname Lucky Imoize, Taiwo Oyedare, Chibuike Gerald Ezekafor, and Sachin Shetty; Deployment of an Energy Efficient Routing Protocol for Wireless Sensor Networks Operating in a Resource Constrained Environment, Transactions on Networks and Communications, Volume 7 No. 1, February (2019); pp: 34-50

well as common classification of routing protocols for WSN. Section IV covers proposed hierarchical routing technique via a detailed description of methodology of the model, parameters used and their descriptions, assumptions, mathematical expression of model and employed algorithm. Focus was more on the Hierarchical-based routing technique using the LEACH approach for healthcare application. Section $\mathrm{V}$ presents the simulations, graphical and tabulated results and indications. Section VI concludes the paper, states useful contributions and recommendations for future studies.

\section{Related work}

Wireless sensor networks have various applications and characteristics. However, the main tasks of the nodes that make up these networks involves sensing and collecting, processing and transmitting data to a site where it is required for either tracking or monitoring functions [7].

Effective power management is a major focus while designing protocols for WSN. There have also been efforts in the recent technology to reduce power consumption of sensor node by local processing [8], which means that sensor nodes now have the capacity to process information more faster before transmission. This has rather increased the circuitry complexity and does not relatively reduce power consumption. Though the average power consumption of wireless sensor devices has exhibited a downward trend over the decades, battery power innovation have not followed this trend. Hence, the smart techniques to consume and optimize power in wireless sensor network is crucial.

Gupta et al. [9] provided the H-LEACH, a typical hybrid system that uses fixed clusters and dynamic cluster head $(\mathrm{CH})$. In their simulation, the authors did not consider key aspects such as energy consumption by the cluster head based on the fact that they were dynamically chosen and would account for a negative impact on the information sent to the base station.

Heinzelman et al. [10], reported a proposal based on the asumption that the entire sensor nodes are potentially taken as base stations. Hence, all nodes disseminate information to other nodes across the network. Rumor routing as established by Braginsky et al. [12] is suited for applications where geographical routing can be very difficult. Zabin et al. [13] reported a proposal on energy aware routing and the reliable, energy efficient protocol (REEP). The later is a new energy awareness routing. Generally, REEP enables sensor nodes to quickly establish more reliable and efficient paths for data transmission and this increases network lifetime.

Heienzelman et al. [11] noted that Low Energy Adaptive Clustering Hierarchy (LEACH) protocol is one of the popular algorithms for WSN. Here, clusters of sensor nodes formed are focused on the received signal quality and the use of a local $\mathrm{CH}$ as a relay or backbone to the base station was presented. Rather than every individual sensor node sending packet to the $\mathrm{BS}$, only a designated $\mathrm{CH}$ node will perform this action at any given round and this greatly reduces energy consumption in a way similar to [14].

Stationary and mobile wireless sensor applications both have diverse challenges and concerns but they share these concerns in common; transmission power, data storage and aggregation capacity, computational capacities and energy consumption [8]. Effective Power management is a major focus while designing protocols for WSN. In this study, the proposed Hierarchical (LEACH) protocol is employed to optimize the energy efficiency of a WSN in a healthcare environment. 


\section{Theoretical background}

\section{A. Routing Protocol Challenges and Design Issues}

Though the emergence of WSN has transformed various aspect of today's technology, there are various challenges and limitations [15] which are consistently reassessed and investigated with the hope of improving functionality and quality of service. Energy supply bandwidth limitation for the links and computational storage capacity will always be areas of research. Improving longevity and lifetime of a WSN network is a priority. To design routing techniques that will cater for these limitations, we need to put to bare, these few challenging factors discussed as follows:

1) Network Dynamics: In earlier applications of sensor nodes, it was always considered that nodes are stationary. For this architectural condition, challenge is minimal when compared with more recent applications where mobility is as important as sensing, for example, health care and wildlife monitoring. It is more challenging to route and consume less energy in mobile WSN. Whether we are talking about tracking or monitoring, the network is dynamic and such requires constant updates and message alerts to keep to the node and environmental requirements. This is a task that requires smart routing protocol.

2) Transmission Media: In mobile sensor network, Multi-hopping is expected. Multi-hopping is done via a wireless link. There is a need to address the issues inherent in wireless channels that could negatively impair performance of the sensor node. In the design of such networks, there is a need to consider the use of Time Division Multiple Access (TDMA) for the Media access protocol. This helps greatly in conserving the energy of the network when compared with the Code Division Multiple Access (CDMA).

3) Node Deployment: Deployment of sensor nodes can be a deterministic or randomized process and this as well affect the performance of protocols of choice. In either case, sensors are manually positioned and the transmission route is predetermined or positions are randomized making it possible to determine routes. The experience creates an ad-hoc infrastructure, which greatly poses challenges in coverage and optimization of clustering.

4) Fault Tolerance: When a sensor node fails, the entire activities of the sensor network, especially when such failure is caused by power depletion, physical damage or environmental constraints, should not be affected. In scenarios when this failure occurs, there must be a MAC and routing protocols that should cater for raising new links and connections as an alternative to keep the network activities alive especially collating data to the base stations. What this means is that multiple redundancy of paths and task channel should always be considered to improve on fault handling capacity of a mobile sensor network.

5) Quality of Service: In most mobile WSN applications like healthcare, there is time limit for data aggregation and transmission to the base station. In this scenario, the moment sensed data is not relayed to the base station for onward processing; data becomes invalid after a particular amount of time. In some applications, the network lifetime and power conservation is of much importance than the integrity of data. This implies that we need to consider the protocol that will suit each kind of application.

6) Coverage: Area coverage of the sensor nodes is of great importance in WSN design. Coverage in mobile wireless sensor network can either mean the communication coverage or the sensing coverage. For which ever context we consider, the coverage affects the accuracy and range of sensor node performance. This has to be considered as a concern when designing a WSN. 
Agbotiname Lucky Imoize, Taiwo Oyedare, Chibuike Gerald Ezekafor, and Sachin Shetty; Deployment of an Energy Efficient Routing Protocol for Wireless Sensor Networks Operating in a Resource Constrained Environment, Transactions on Networks and Communications, Volume 7 No. 1, February (2019); pp: 34-50

7) Energy Consumption: Since sensor nodes exhaust their energy supply during transmission and computation in WSN setting, it is always important to apply energy conservation techniques and model in network design. Life span of a sensor node is dependent on the battery life as the source of energy [11]. Once depleted and there are no alternative route provision via redundancy, WSN is affected. There is a need to keep an eye on this challenge in design.

\section{B. Classifications of Routing Protocols for WSNs}

Various routing protocols have evolved basically to avert the challenges and poor services experienced in this resource constrained wireless sensor network architecture. Generally, routing protocols can be in flatbased routing [16], Location based routing or Hierarchical based routing [17]. The routing model decided in each design is dependent also on; the type of network structure, the initiator of communication, network operation carried out using this protocol and the type of communication routes processed from source to sink. Routing protocol is flat-based if all sensor nodes within the network are assigned the same role and function. In location based protocols [18], sensor node functionality ties greatly to the position of these nodes in the network, while in hierarchical based routing [18], nodes are seen to carry out various roles in the network based on assigned role and hierarchy.

A routing protocol can be considered to be adaptive if the nodes have the ability to adjust intelligently to existing parameters for proper network and node functioning. In this form of protocol, it can be multipath, query or negotiation based. It can also be QoS based or dependent on the protocol operations. Routing protocols also can be reactive, proactive or hybrid depending on how data routing is established from source to destination. We also have cooperative routing where data aggregates to a central point for further processing before sending to a base station, this is to ensure cost is minimized [19].

\section{Proposed Hierarchical Routing Technique}

There are various methods and techniques used in hierarchical routing. However, the basic concept is that sensor nodes are clustered, and within the formed cluster, a node is picked as the cluster head depending on the amount of energy left within that node. All other nodes then forward their data directly to the selected head. Note however that the cluster head will have its energy depleted faster than any other node in the cluster as it has to send more data to the next cluster or the base station than the other sensor nodes. The position of the cluster head is therefore rotated on every iteration of the simulation loop to keep the residual energy within the sensor nodes balanced.

In a typical health care unit, there will be movement of the patients from one location to the other. Since sensors are attached to a patient, his/her movement causes the position of a sensor node to change relative to its cluster head. If a sensor node is moved out of proximity from its cluster head, it may have to join a new cluster to reduce the energy consumed in sending its packet. If and when the sensor node comes back to its previous location, it rejoins its original cluster. Modeling the change in location of a sensor node however is not a straight forward model as this is statistical in nature. A patient may or may not decide to move from his/her location. This behavior is modeled by creating some locations on the model called "service points" where a patient may need to go every once in a while. The service points are deployed as well as the sensors at the start of the simulation. Randomly, a sensor node is selected and mobilized to a service point, kept in that region for some simulation rounds and then moved back to its original location. 


\section{A. Methodology}

The method involved in modeling the proposed hierarchical routing technique in MATLAB used the object oriented programming (OOP) approach. In this approach, each entity in the model is programmatically abstracted or represented. In this model, the following entities are identified: the BS, the WSN, the cluster, servicing point and the ward.

1) The Base Station (BS): Every data acquired by the WSN is assumed to be destined for a data center meant to aggregate such data, process them and could be stored in memory for further re-transmission. The data center is described in the model as the base station. However, note that the base station is modeled differently from the wireless sensor node even though they share some similarities such as the ability to receive data from a neighboring WSN. The different model used is to account for the fact that the base station energy cannot be depleted given that the BS is grid powered. In the model, the base station has these properties; Location and packet received. Location considers the $X$ and $Y$ coordinate positions for the BS while the packet received depicts the set of data that has been relayed or logged at the base station.

2) Wireless Sensor Node (WSN): A sensor unit consists of the sensor and the analogue to digital converter circuitry. Generally, sensors collect physical data and convert to an electrical signal. This signal is in a format that can be interpreted by other processing units.

Here, it should not be assumed that the location is fixed during the cause of this simulation; this means a patient may relocate to a new vector coordinate on the simulation space. The sensor node model has the following properties; energy, location, whether dead or not, and packet to transmit.

During simulation, the residual energy of a sensor node is always re-estimated every time a sensor receives or sends a data packet (DP). If residual energy (RE) within such sensor is not enough to receive the incoming packet or send outgoing packet, the sensor is marked as dead and will no longer participate in the network. The incoming or outgoing packet is therefore lost. Wireless Sensor Nodes are also mobile in this case. This has an implication on the geographical location of the WSN during this simulation. When a sensor moves, chances are that it will no longer be in proximity to the current cluster to which it belongs. The sensor will therefore be detached from its current cluster and attached to another. In reality, the selection of which sensor to move and the location of a sensor node when it moves is statistical in nature as there is no way to programmatically define which sensor moves and the new location of this sensor node when it moves. To circumvent this problem, sensors are selected randomly using a random number generator. The location of the Service Center (SC) to mobilize the sensor node to is also randomized. The selected sensor is therefore relocated to the SC, kept for a number of simulation rounds and finally moved back to its original location.

3) The Cluster: The intended optimization to be performed in this study is meant to be accomplished using cluster formation. A cluster is simply a group of wireless sensor node in proximity to each other. Clusters are formed geographically by dividing the simulation area into some banding rectangles. Sensor nodes falling within the same banding rectangle are assigned to a common cluster. After cluster formation, a cluster head $(\mathrm{CH})$ election is performed using a cost function as described in later part of this section. The sensor node with the minimum cost is delegated as $\mathrm{CH}$. Due to the dynamic position of sensor nodes, $\mathrm{CH}$ election is repeated on every simulation round as the $\mathrm{CH}$ itself may have migrated away from the cluster 
Agbotiname Lucky Imoize, Taiwo Oyedare, Chibuike Gerald Ezekafor, and Sachin Shetty; Deployment of an Energy Efficient Routing Protocol for Wireless Sensor Networks Operating in a Resource Constrained Environment, Transactions on Networks and Communications, Volume 7 No. 1, February (2019); pp: 34-50

leaving the cluster without a head. Therefore, the election is performed over and over again to establish or ascertain that there will be a head within the cluster.

4) The Service Center (SC): This makes provision for the mobility of the sensor nodes. Service centers are strategically positioned at the beginning of the simulation and they define certain locations that a patient with a WSN attached to may have to migrate to once in a while. The number of patients that are mobilized to service center is a pre-determined value in this paper.

5) The Ward: Ward as used here is a hospital location where patients are cared for specially. This is always made for patients on admission. Patients with various levels of attention are allocated to various wards. It can also be based on gender, age or treatment type. In this paper, our wards are modeled to have patients with wearable or implanted wireless sensor nodes which are grouped based on zones or clusters.

\subsection{System Energy Model of a Sensor Node}

As stated in [20], the energy suitable for a sensor node to transmit its packets to a base station is a function of the number of data in the packet, the distance separating the sensor node from the destination of the packet is as shown in figure 1

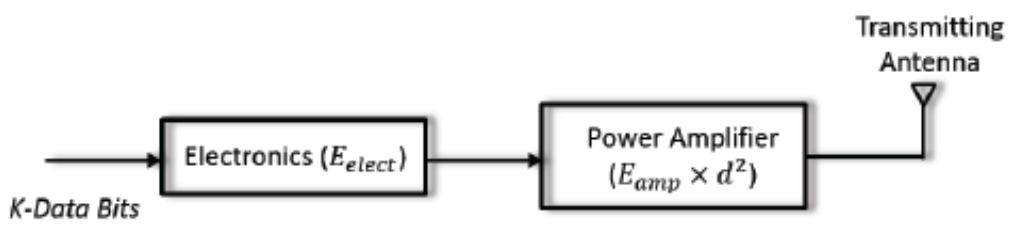

Figure. 1. Energy model diagram for a sensor node (sending)

where $k$ stands for the number of data bits to be transmitted from a sensor node.

$E_{\text {elect }}$ is energy required by the electronics to send one data bit.

$E_{a m p}$ is energy required by the power amplifier to send one data bit through a distance of $1 \mathrm{~m}$.

$d$ represents the separation distance between the transmitter and the receiver.

The total energy required to send the k-data bits is mathematically expressed in equation 1

$$
E_{t x}=\left(E_{\text {elect }} \times k\right)+\left(E_{a m p} \times d^{2} \times k\right)
$$

While receiving a packet by a cluster head, an amount of energy is expended in receiving the packet. This is denoted as in figure 2 . This is mathematically expressed as shown in (2)

$$
E_{r x}=\left(E_{\text {elect }} \times k\right)
$$

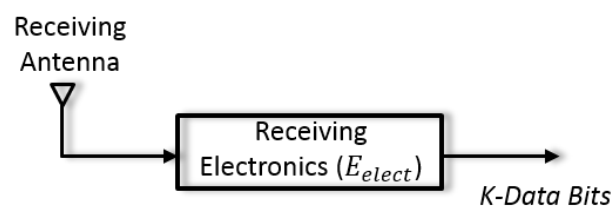

Fig. 2: Energy model diagram for a sensor node (receiving) 


\subsection{The Proposed Routing Protocol Algorithm}

The proposed routing algorithm focuses on extending the life-cycle of the network. First, sensor nodes are geographically formed into clusters depending on the proximity of the nodes to each other. This is done by breaking down the work area in to a specific number of rectangles. Sensor nodes falling into the same rectangle are grouped together to form a cluster, and the cluster head is then chosen depending on the amount of energy left within the sensor node and the distance between the sensor node and the base station. This is to limit the possibility of the data being sent from a sensor node traveling back and forth. The selection of the cluster head therefore is a function of two variables, henceforth denoted as cost. The cost function is expressed in equation 3.

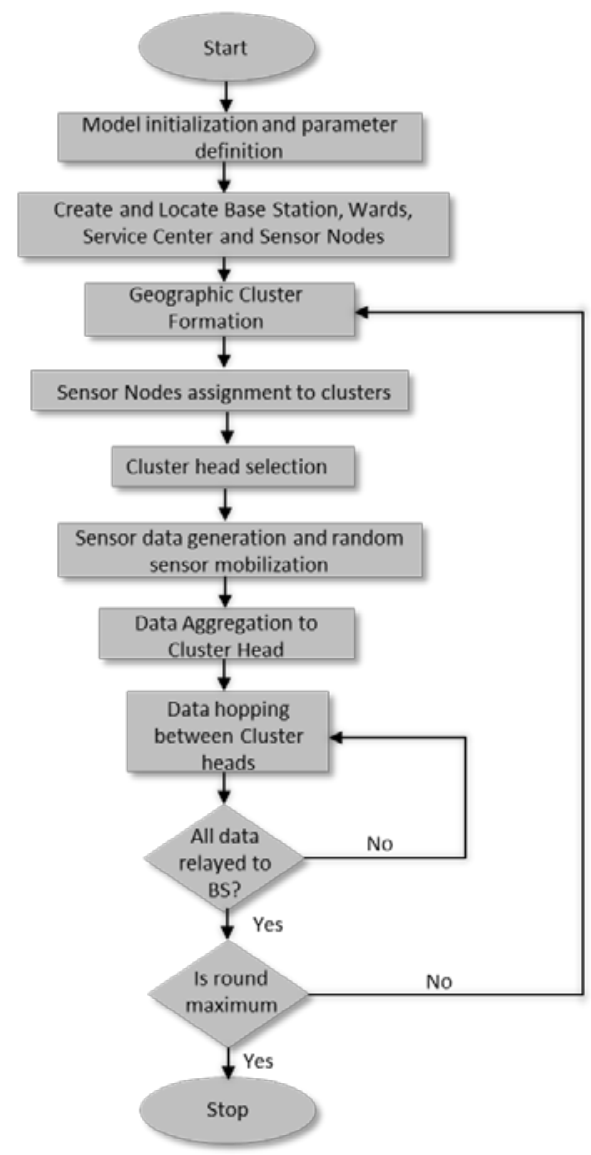

Fig. 3: A typical algorithm for the Hierarchical based routing model

$$
E_{\text {cost }}=\left(E_{i}-E_{s}\right) \times W_{s}+d_{b s} \times W_{b}
$$

where $E_{\text {cost }}$ is the cost function

$E_{i}$ represents initial energy assigned to all sensor nodes

$E_{s}$ represents energy residue within a sensor node

$W_{s}$ represents a weight factor for the energy residue

$W_{b}$ represents a weight factor for the energy consumed $d_{b s}$ represents separation distance between a sensor and the BS. 
Agbotiname Lucky Imoize, Taiwo Oyedare, Chibuike Gerald Ezekafor, and Sachin Shetty; Deployment of an Energy Efficient Routing Protocol for Wireless Sensor Networks Operating in a Resource Constrained Environment, Transactions on Networks and Communications, Volume 7 No. 1, February (2019); pp: 34-50

The cost is then computed for all the sensor nodes within a cluster and the one with the minimal cost is chosen to be the cluster head. When the head has been selected, all sensor nodes aggregate their data to the head.

For the data to reach its destination at the BS, a multihop approach was used where the data get routed to where the data is routed through the closest cluster head until it arrives at the base station.

While routing the data however to the next cluster head, a predictive function should be established as there may be a number of clusters that the data can be channeled through. The forward prediction establishes the amount of power that will be consumed if the data is channeled through a particular branch in the cluster tree. Starting from the root of the tree, a walk is successively made from the root to the base station through different paths and the energy consumed is recorded. At the end of the walk, the path that consumes the minimum energy is taken and the data is forwarded to the next cluster head in that path.

The algorithm followed by the simulation is summarized as; ward positioning, service center location, sensor node deployment, cluster formation, cluster head selection, data aggregation and data multihopping.

1) Ward Positioning: The positioning of the ward used in this simulation is towards the sides of the simulation area/landmark chosen.

2) Service Center Location: A patient may need to migrate from his/her ward for one reason or the other and this is based on the assumption that the sensor node is attached to the body of the patient. This migration causes the location of the sensor node to change. The sensor detaches itself from a cluster and joins another one. When the patient resume back to his ward, the sensor node rejoin its initial cluster.

3) Sensor Node Deployment: At the start of the simulation, sensors are created and positioned on the simulation field. Each sensor node is also assigned an equal amount of energy. The location of the sensor is done in a manner that reflects a real world scenario where patients are expected to be assigned into a ward. Sensor nodes are deployed on a per-ward-basis with a particular amount of sensor nodes in a ward.

4) Cluster Formation: Sensor nodes are always grouped in to clusters to conserve the energy consumed by the network. The cluster formation algorithm is derived on the basis of the geographical location of a sensor node. Due to the mobility of the patient however, cluster grouping is repeated on every simulation cycle as a sensor may have moved away from its last known location. To find an optimum point for the network, the clusters' geographical area is varied between simulations. Increasing this area causes more sensor nodes to fall within a particular area and vice versa.

5) Cluster Head Selection: As stated earlier, the cluster head is elected based on a cost function. The sensor node with a minimum cost gains the position of a cluster head.

6) Data Aggregation: At the onset of every simulation round, a specific amount of data packet is assigned for each sensor node to transmit. After cluster head election, the entire sensor nodes within the cluster send their packets down to the cluster head. At this stage, the cluster head does not send any data, but rather merges the incoming packet with its own data packet. 
7) Multi-Hopping: After the aggregation stage, every cluster head has to send its packet and its children packet to the next cluster head nearest to the base station. The packets then hop from one cluster head to the next until the base station is reached. The cluster head to hop data to is also selected based on a tree walk from the cluster head to the base station via many routes. The route with the minimum power requirement is taken. Figure 3 shows the algorithm for the model.

\section{Results and Discussion}

In the previous section, an improved LEACH protocol for mobile wireless sensor node was proposed. Here we provide a simulation of our improved protocol and estimate the performance of the protocol under varying simulation conditions. There are varieties of tools that could have been used for this modeling, but the environment used for the simulation is MATLAB. This program was chosen as the preferred language because of its availability and ease of use.

\section{A. Simulation Setup and Scenarios}

In this simulation, a health care environment layout was created with a dimension of $400 \mathrm{~m}$ by $400 \mathrm{~m}$. Within this boundary, the base station is positioned at the center with a vector coordinate of 200, 200. Followed by this is positioning the service centers, wards and finally sensor nodes within the wards. Table 1 shows the simulation parameters used in this study.

Simulation was performed by varying the cluster grouping of the network. Clusters were formed geographically by dividing the working space vertically and horizontally into different sizes starting from 1 to 15 . For example, when forming clusters with two groups per length and two groups per breadth, the environment looks like figure 4.

The description of the symbols in figure 4 is explained briefly as follows:

i. $\quad$ Cluster group formations are marked with Purple

ii. Base Station marked in Cyan Dots and Circles

iii. Service centers marked with Cyan Lines

iv. Wards marked with Blue Lines

v. Sensor Nodes marked with Red Dots

vi. Cluster Heads marked with Red Circular Dots

Figure 4 indicates the geographic formation of the cluster. Sensor Nodes falling within the same cluster box are automatically placed in the same cluster. Note however that even if sensors fall within the same ward, and are divided into different cluster by a cluster line, they will be placed in different clusters as depicted in figure 5 . 
Agbotiname Lucky Imoize, Taiwo Oyedare, Chibuike Gerald Ezekafor, and Sachin Shetty; Deployment of an Energy Efficient Routing Protocol for Wireless Sensor Networks Operating in a Resource Constrained Environment, Transactions on Networks and Communications, Volume 7 No. 1, February (2019); pp: 34-50

Table I: List of Defined Parameters and Values Used in Simulation

\begin{tabular}{|c|c|}
\hline Parameter & Value \\
\hline Landmark Length & $400 m$ \\
\hline Landmark Breadth & $400 m$ \\
\hline Number of Base Station & 1 \\
\hline Base Station Location & {$[200,200]$} \\
\hline Number of Service centers & 2 \\
\hline $\begin{array}{l}\text { Service Center } \\
\text { Locations }[X, Y, L, B]\end{array}$ & {$[[252005050][3752005050]]$} \\
\hline Number of Wards & 16 \\
\hline Wards Location $[X, Y, L, B]$ & 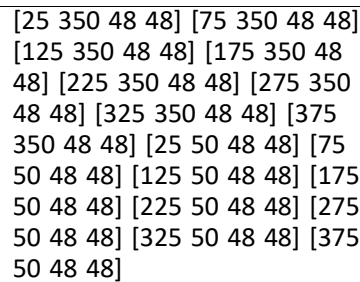 \\
\hline Initial Sensor Energy & $10 \mathrm{~J}$ \\
\hline $\begin{array}{l}\text { Transmitter Electronics Energy } \\
\text { Per bit Etx }\end{array}$ & $5 n J$ \\
\hline $\begin{array}{l}\text { Transmitter Electronics Energy } \\
\text { Per bit Erx }\end{array}$ & $5 n J$ \\
\hline $\begin{array}{l}\text { Transmitter Power Amplifier } \\
\text { Per bit }\end{array}$ & $5 n J$ \\
\hline Maximum Simulation Rounds & 1500 \\
\hline Sensors per Ward & 5 \\
\hline Maximum Sensors on Transit & 5 \\
\hline Maximum Sensor Speed & $1 \mathrm{~m} / \mathrm{s}$ \\
\hline Sensor Data packet size & 2000 bits \\
\hline Cluster Formation Range & $1: 15$ \\
\hline
\end{tabular}

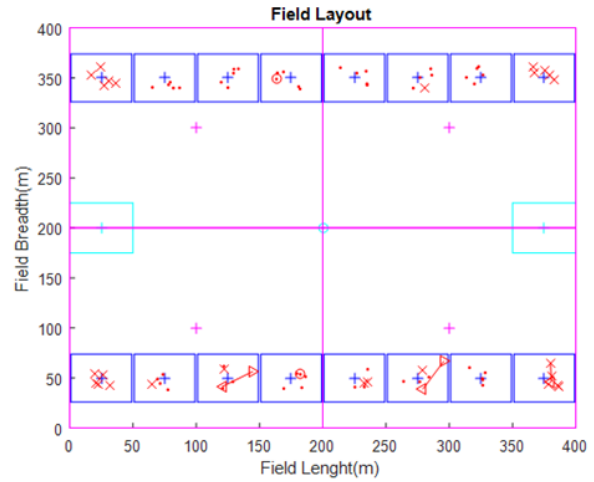

Fig. 4: A Sample $2 \times 2$ cluster field layout for defined $400 \mathrm{~m} \times 400 \mathrm{~m}$ Area

Sensors drawn in triangular symbols above as shown in figure 4 and 5 indicate mobile sensors currently on transit to or from a service center. For each simulation rounds, the energy left within each sensor is estimated and when a sensor does not have enough energy to send its data, it is marked dead as depicted in figure 6.

Figure 6 shows the conclusive layout of a simulation where all sensors are dead as marked with an ' $x$ '. At the end of each simulation, the network lifetime of the system is estimated from a graph depicting the number of active nodes plotted against the simulation rounds in figure 7. 


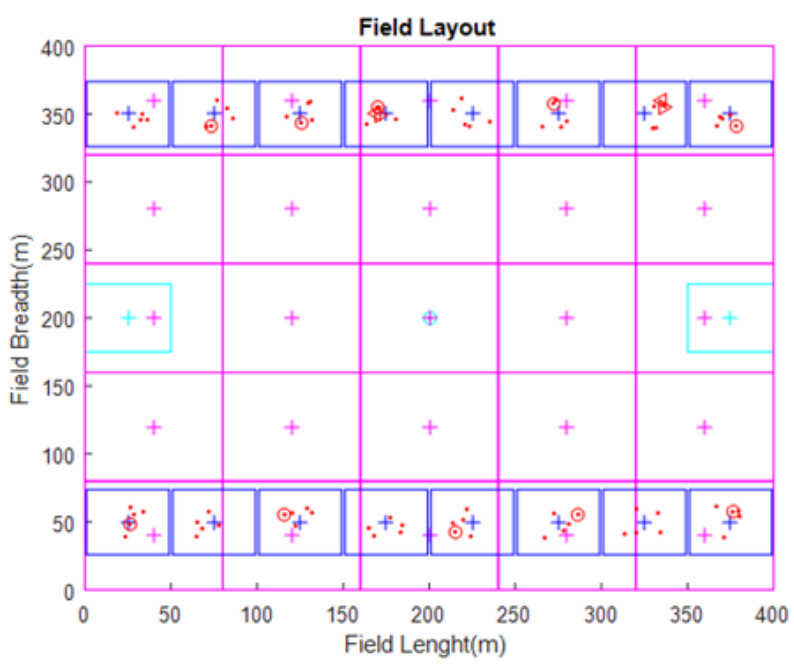

Fig. 5: Sample 5x5 cluster formation with a ward mapped to different cluster/zone

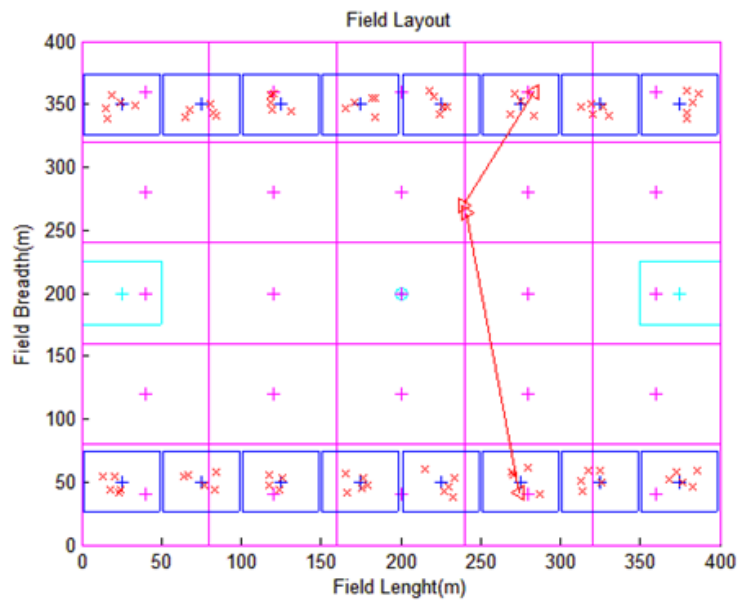

Fig. 6: Sample $5 \times 5$ cluster depicting dead sensors

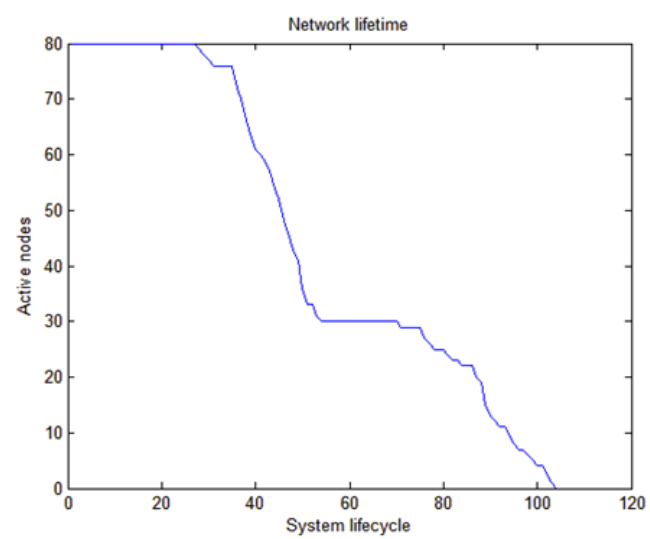

Fig. 7: Sample of a network lifetime graph indicating active nodes per round

\section{B. Simulation Results}

1) Combined Network Lifetime: Having repeated the stated procedure, some of the graphs for the cluster groups between non-hierarchical and hierarchical are generated and presented in figures 4 - 6 . It should 
Agbotiname Lucky Imoize, Taiwo Oyedare, Chibuike Gerald Ezekafor, and Sachin Shetty; Deployment of an Energy Efficient Routing Protocol for Wireless Sensor Networks Operating in a Resource Constrained Environment, Transactions on Networks and Communications, Volume 7 No. 1, February (2019); pp: 34-50

be noted that in order to present brief results, we show cluster results for only $2 \times 2$ and $5 \times 5$ clusters. It is important to note that we see similar trends for other cluster sizes.

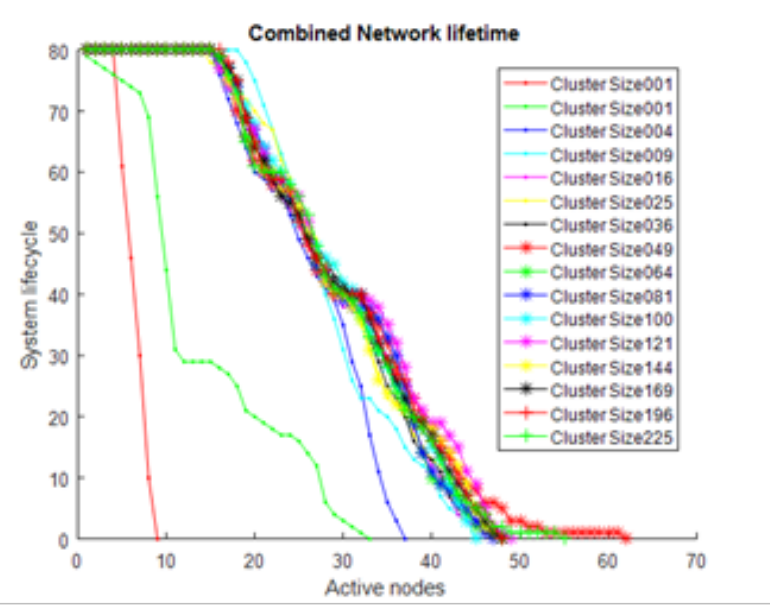

Fig. 10: Combined graphical network lifetime for considered routing protocols and cluster formation

From figure 10, it can be deduced that the "First-to-die" sensor time of the system is relatively proportional to the cluster groups. In the non-hierarchical network, the first sensor is observed dead at round $x x$. While in the hierarchical network, this time increases considerably. All these can be observed in table II:

The cluster formation that gave the best network lifetime was observed at $6 \times 6$ hierarchical cluster division. It should be noted, however, that the results obtained in this study are only applicable for the layout used. A change in the environment layout may definitely give a new result entirely. The results obtained here should therefore not be taken as a generic solution applicable to other instances.

Table II: Statistical average round for non-hierarchical and hierarchical orders

\begin{tabular}{|c|c|c|c|c|c|}
\hline $\begin{array}{c}\text { Routing } \\
\text { Protocol/O } \\
\text { rder }\end{array}$ & Clustering & $\begin{array}{c}\text { First- } \\
\text { To-Die } \\
\text { Round }\end{array}$ & $\begin{array}{c}\text { Active } \\
\text { node } \\
\text { life time } \\
\text { at 50\% }\end{array}$ & $\begin{array}{c}\text { Last-to-die } \\
\text { sensor round }\end{array}$ & $\begin{array}{c}\text { Statistical } \\
\text { Average } \\
\text { weight of } \\
\text { rounds }\end{array}$ \\
\hline N.H.O. & 0 & 4 & 6 & 9 & 6.3 \\
\hline H.O. 1 & $1 \times 1$ & 2 & 10 & 32 & 14.7 \\
\hline H.O. 4 & $2 \times 2$ & 15 & 29 & 40 & 28 \\
\hline H.O. 9 & $3 \times 3$ & 17 & 27 & 46.5 & 30.2 \\
\hline H.O. 16 & $4 \times 4$ & 15 & 26 & 46.5 & 29.2 \\
\hline H.O. 25 & $5 \times 5$ & 16 & 30 & 47 & 31 \\
\hline H.O. 36 & $6 \times 6$ & 15 & 32 & 54 & 33.7 \\
\hline H.O. 49 & $7 \times 7$ & 16.5 & 34 & 47.5 & 32.7 \\
\hline H.O. 64 & $8 \times 8$ & 15 & 32 & 48 & 31.7 \\
\hline H.O. 81 & $9 \times 9$ & 15 & 29 & 46 & 30 \\
\hline $\begin{array}{c}\text { H.O. } \\
100\end{array}$ & $10 \times 10$ & 15 & 32 & 46.5 & 31.2 \\
\hline $\begin{array}{c}\text { H.O. } \\
121\end{array}$ & $11 \times 11$ & 15 & 28 & 50 & 31 \\
\hline $\begin{array}{c}\text { H.O. } \\
144\end{array}$ & $12 \times 12$ & 15 & 29 & 46 & 30 \\
\hline $\begin{array}{c}\text { H.O. } \\
169\end{array}$ & $13 \times 13$ & 14.5 & 27 & 47 & 29.5 \\
\hline $\begin{array}{c}\text { H.O. } \\
196\end{array}$ & $14 \times 14$ & 14.5 & 27 & 48 & 29.8 \\
\hline $\begin{array}{c}\text { Hierarchic } \\
\text { order } 225\end{array}$ & al $15 \times 15$ & 14.5 & 30 & 50 & 31.5 \\
\hline O. & & & & & \\
\hline
\end{tabular}

N.H.O. = Non Hierarchical Order; H.O. = Hierarchical Order 
Table III shows a comparison between previous approaches and the approach followed in this paper. First, a comparison is made between the proposed model and the models reported in [11] and [14]. The statistical average weight of rounds in [14]; for non-hierachical is 300, 1115 for the hierarchical LEACH comprising of 5 cluster heads, and 3.7 for the round increase ratio. For [11], the round first node dies is 217 , and the round last node dies is 468 , and the statistical average weight of rounds is 451 , for the nonhierarchical. Here, hierarchical LEACH with 5 cluster heads show a round first node dies of 1848 and round last node dies of 2609, with a statistical average weight of rounds increase ratio of 4.9. Finally, the proposed LEACH with 6 cluster heads reveal 1583 round first node dies, 2793 for the round last node dies, and 2188 statistical average weight of rounds. Here, results show that the proposed model agree closely with the existing models presented in [11] and [14].

Table III: Combining and comparing results

\begin{tabular}{|c|c|c|c|c|}
\hline $\begin{array}{l}\text { Simulation/ } \\
\text { Model Type }\end{array}$ & $\begin{array}{l}\text { Routing } \\
\text { Protocol }\end{array}$ & $\begin{array}{c}\text { Round } \\
\text { First } \\
\text { node dies }\end{array}$ & $\begin{array}{c}\text { Round } \\
\text { Last node } \\
\text { dies }\end{array}$ & $\begin{array}{l}\text { Statistical } \\
\text { Average } \\
\text { weight of } \\
\text { rounds }\end{array}$ \\
\hline \multirow[t]{3}{*}{$\begin{array}{c}\text { Yadav model } \\
{[14]}\end{array}$} & $\begin{array}{c}\text { Non- } \\
\text { Hierarchical }\end{array}$ & 120 & 240 & 300 \\
\hline & $\begin{array}{c}\text { Hierarchical } \\
\text { (LEACH) } \\
\text { (5 Cluster } \\
\text { Heads) }\end{array}$ & 930 & 1300 & 1115 \\
\hline & $\begin{array}{l}\text { Round in- } \\
\text { crease } \\
\text { ra-tio }\end{array}$ & 7.8 & 5.4 & 3.7 \\
\hline \multirow{3}{*}{$\begin{array}{c}\text { Own } \\
\text { simulation } \\
\text { using model } \\
{[14]+\text { mobile }} \\
\text { sensors }\end{array}$} & $\begin{array}{c}\text { Non- } \\
\text { Hierarchical }\end{array}$ & 35 & 74 & 54.5 \\
\hline & $\begin{array}{c}\text { Hierarchical } \\
\text { (LEACH) } \\
\text { (3 Cluster } \\
\text { Heads) }\end{array}$ & 712 & 1793 & 1252.5 \\
\hline & $\begin{array}{l}\text { Round in- } \\
\text { crease } \\
\text { ra- tio }\end{array}$ & 20.3 & 24.2 & 22.98 \\
\hline \multirow{3}{*}{$\begin{array}{l}\text { Heinzelman } \\
\text { model [11] }\end{array}$} & $\begin{array}{c}\text { Non- } \\
\text { Hierarchical }\end{array}$ & 217 & 468 & 451 \\
\hline & $\begin{array}{c}\text { Hierarchical } \\
\text { (LEACH) } \\
\text { (5 Cluster } \\
\text { Heads) }\end{array}$ & 1848 & 2609 & 2228.54 \\
\hline & $\begin{array}{l}\text { Round in- } \\
\text { crease } \\
\text { ra- tio }\end{array}$ & 8.5 & 5.6 & 4.9 \\
\hline \multirow{3}{*}{$\begin{array}{c}\text { Own } \\
\text { simulation } \\
\text { using model } \\
{[11]+\text { mobile }} \\
\text { sensors }\end{array}$} & $\begin{array}{c}\text { Non- } \\
\text { Hierarchical }\end{array}$ & 57 & 131 & 94 \\
\hline & $\begin{array}{c}\text { Hierarchical } \\
\text { (LEACH) } \\
\text { (6 Cluster } \\
\text { Heads) }\end{array}$ & 1583 & 2793 & 2188 \\
\hline & $\begin{array}{l}\text { Round in- } \\
\text { crease } \\
\text { ra- tio }\end{array}$ & 27.8 & 21.32 & 23.7 \\
\hline \multirow{3}{*}{$\begin{array}{c}\text { Own Visual } \\
\text { Studio Life/ } \\
\text { validation } \\
\text { model results }\end{array}$} & $\begin{array}{c}\text { Non- } \\
\text { Hierarchical }\end{array}$ & 6 & 108 & 56 \\
\hline & $\begin{array}{c}\text { Hierarchical } \\
\text { (LEACH) } \\
\text { (1 Cluster } \\
\text { Heads) }\end{array}$ & 89 & 128 & 172.5 \\
\hline & $\begin{array}{l}\text { Round in- } \\
\text { crease } \\
\text { ra-tio }\end{array}$ & 14.8 & 1.2 & 3.08 \\
\hline
\end{tabular}


Agbotiname Lucky Imoize, Taiwo Oyedare, Chibuike Gerald Ezekafor, and Sachin Shetty; Deployment of an Energy Efficient Routing Protocol for Wireless Sensor Networks Operating in a Resource Constrained Environment, Transactions on Networks and Communications, Volume 7 No. 1, February (2019); pp: 34-50

\section{Conclusion and Future Work}

\section{A. Conclusion}

This study has shown that quality of service, cost of maintenance and replacements of energy sources, healthcare giver efficiency can be greatly improved by implementing Hierarchical (LEACH) routing protocol. Cluster formation will be dependent on the size of locations in question and application. Choice of simulation parameters, locations of sensor nodes and base stations as well as other factors will always impact on results as seen in the comparison tables shared in section $\mathrm{V}$ of this paper.

In this study, results show that hierarchical routing technique based on low energy adaptive clustering protocol increased the energy efficiency of WSN for the predetermined simulation condition. It was shown that clustering and hierarchical routing techniques greatly improve the network lifetime and depending on the number of clusters formed, the network lifetime varies. It was observed however that the rise in the network lifetime with more clusters stopped at certain cluster level after which further increase in the cluster numbers resulted in either no increase in network lifetime or a further decrease.

\section{B. Future Work}

Future studies could use a practical healthcare facility layout as the basis for simulation. In this paper, we did not consider the case where patient wards are located in structures with more than one floor. Therefore, it is recommended that future works put this into consideration. Furthermore, in order to limit the amount of energy expended in sending data packets, these packets may be compressed prior to transmission. However, if the compression algorithm used consumes a noticeable amount of power, it should be carefully investigated.

\section{Miscellaneous - Conflict of Interest Declaration}

The authors declare that there is no conflict of interest regarding the publication of this paper.

\section{REFERENCES}

[1] D. Shinghal, N. Srivastava, et al., "Wireless sensor networks in agriculture: for potato farming," 2017.

[2] B. Wang, X. Gu, L. Ma, and S. Yan, "Temperature error correction based on bp neural network in meteorological wireless sensor network," International Journal of Sensor Networks, vol. 23, no. 4, pp. 265-278, 2017.

[3] Gauravpaliwal and Pankajkasar, "Article: Wireless body area net- work for ubiquitous mhealth mobile patient monitoring systems: Architecture, opportunities and challenges," IJCA Proceedings on National Conference on Emerging Trends in Computer Technology, vol. NCETCT, pp. 1-6, December 2014.

[4] M. H. Anisi, G. Abdul-Salaam, M. Y. I. Idris, A. W. A. Wahab, and Ahmedy, "Energy harvesting and battery power based routing in wireless sensor networks," Wireless Networks, vol. 23, no. 1, pp. 249-266, 2017. 
[5] K. Fang, C. Liu, and J. Teng, "Cluster-based optimal wireless sensor deployment for structural health monitoring," Structural Health Monitoring, vol. 17, no. 2, pp. 266-278, 2018.

[6] B. Lo and G.-Z. Yang, "Body sensor networks-research challenges and opportunities," 2007.

[7] C. Umamaheswari, J. Gnanambigai, et al., "Energy optimization in wireless sensor network using sleep mode transceiver," Global Journal of Research In Engineering, vol. 11, no. 3, 2011.

[8] J. Stankovic, Q. Cao, T. Doan, L. Fang, Z. He, R. Kiran, S. Lin, S. Son, R. Stoleru, and A. Wood, "Wireless sensor networks for in- home healthcare: Potential and challenges," in High confidence med- ical device software and systems (HCMDSS) workshop, vol. 2005, 2005.

[9] V. Gupta and M. Doja, "H-leach: Modified and efficient leach protocol for hybrid clustering scenario in wireless sensor networks," in Next-Generation Networks, pp. 399-408, Springer, 2018.

[10] J. Kulik, W. Heinzelman, and H. Balakrishnan, "Negotiation-based protocols for disseminating information in wireless sensor net- works," Wireless networks, vol. 8, no. 2/3, pp. 169-185, 2002.

[11] W. R. Heinzelman, A. Chandrakasan, and H. Balakrishnan, "Energy-efficient communication protocol for wireless microsensor networks," in System sciences, 2000. Proceedings of the 33rd annual Hawaii international conference on, pp. 10-pp, IEEE, 2000.

[12] D. Braginsky and D. Estrin, "Rumor routing algorthim for sensor networks," in Proceedings of the 1st ACM international workshop on Wireless sensor networks and applications, pp. 22-31, ACM, 2002.

[13] F. Zabin, S. Misra, I. Woungang, H. F. Rashvand, N.-W. Ma, and M. A. Ali, "Reep: data-centric, energy-efficient and reliable routing protocol for wireless sensor networks," IET communications, vol. 2, no. 8, pp. 995-1008, 2008.

[14] L. Yadav and C. Sunitha, "Low energy adaptive clustering hierarchy in wireless sensor network (leach)," International journal of com- puter science and information technologies, vol. 5, no. 3, pp. 4661-4664, 2014.

[15] R. S. Istepanian, E. Jovanov, and Y. Zhang, "Guest editorial introduction to the special section on m-health: Beyond seamless mobility and global wireless health-care connectivity," IEEE Transactions on information technology in biomedicine, vol. 8, no. 4, pp. 405-414, 2004.

[16] R. C. Shah and J. M. Rabaey, "Energy aware routing for low energy ad hoc sensor networks," in Wireless Communications and Networking Conference, 2002. WCNC2002. 2002 IEEE, vol. 1,pp. 350-355, IEEE, 2002.

[17] J. N. Al-Karaki and A. E. Kamal, "Routing techniques in wireless sensor networks: a survey," IEEE wireless communications, vol. 11, no. 6, pp. 6-28, 2004. 
Agbotiname Lucky Imoize, Taiwo Oyedare, Chibuike Gerald Ezekafor, and Sachin Shetty; Deployment of an Energy Efficient Routing Protocol for Wireless Sensor Networks Operating in a Resource Constrained Environment, Transactions on Networks and Communications, Volume 7 No. 1, February (2019); pp: 34-50

[18] K. Akkaya and M. Younis, "A survey on routing protocols for wireless sensor networks," Ad hoc networks, vol. 3, no. 3, pp. 325-349, 2005.

[19] S. Dai, X. Jing, and L. Li, "Research and analysis on routing pro- tocols for wireless sensor networks," in Communications, Circuits and Systems, 2005. Proceedings. 2005 International Conference on, vol. 1, pp. 407-411, IEEE, 2005.

[20] W. R. Heinzelman, J. Kulik, and H. Balakrishnan, "Adaptive proto- cols for information dissemination in wireless sensor networks," in Proceedings of the 5th annual ACM/IEEE international conference on Mobile computing and networking, pp. 174-185, ACM, 1999. 